\title{
Experimental and Numerical Study of Cervical Muscle Contraction in Frontal Impact
}

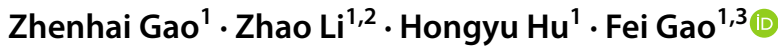

Received: 4 March 2019 / Accepted: 19 April 2019 / Published online: 24 May 2019

(c) The Author(s) 2019

\begin{abstract}
In a crash situation, drivers typically make evasive maneuvers before an upcoming impact, which can affect the kinematics and injury during impact. The purpose of the current study was to investigate the response and effect of drivers' cervical muscles in a frontal impact. A crash scenario was developed using a vehicle driving simulator, and 10 volunteers were employed to drive the simulator at $20 \mathrm{~km} / \mathrm{h}, 50 \mathrm{~km} / \mathrm{h}, 80 \mathrm{~km} / \mathrm{h}$ and $100 \mathrm{~km} / \mathrm{h}$. Electromyography (EMG) was recorded from the sternocleidomastoideus (SCM), splenius cervicis (SPL) and trapezium (TRP) muscles using a data acquisition system, and the level of muscle activation was calculated. A numerical study was conducted using data collected in the experiment. The results revealed that the cervical muscles were activated during drivers' protective action. EMG activity of cervical muscles before impact was greater than that during normal driving. EMG activity increased with driving speed, with the SCM and TRP exhibiting larger increases than the SPL. The kinematics and load of the driver were influenced by muscle activation. Before the collision, the head of an active model stretched backward, while the passive model kept the head upright. In low-speed impact, the torque and shear of the cervical muscle in the active model were much lower than those in the passive model, while the tension of the cervical muscle was higher in the active model compared with the passive model. The results indicated that the incidence of cervical injury in high-speed impact is complex.
\end{abstract}

Keywords Cervical injury $\cdot$ Frontal impact $\cdot$ Active muscle force $\cdot$ Driving simulator

$\begin{array}{ll}\text { Abbreviations } \\ \text { EMG } & \text { Electromyography } \\ \text { SCM } & \text { Sternocleidomastoideus } \\ \text { SPL } & \text { Splenius cervicis } \\ \text { TRP } & \text { Trapezium } \\ \text { MVC } & \text { Max voluntary contraction } \\ \text { HBM } & \text { Human body model } \\ \text { RMS } & \text { Root mean square } \\ \text { NCAP } & \text { New Car Assessment Programme }\end{array}$

Fei Gao

gaofei123284123@163.com

1 State Key Laboratory of Automotive Simulation and Control, Jilin University, Changchun 130000, China

2 School of Public Health, Jilin University, Changchun 130000, China

3 College of Biological and Agricultural Engineering, Jilin University, Changchun 130000, China

\section{Introduction}

Traffic accidents are one of the leading causes of death worldwide. In 2015, there were 35,092 fatalities and 2,443,000 injuries related to road accidents in the USA [1], and 26,134 and 1,090,042 fatalities and injuries, respectively, in the European Union [2]. In cases of deaths and injuries in road accidents, the head and neck are the most vulnerable parts of the body [3, 4]. Although cervical injuries caused by rear-end collisions have been extensively studied, almost one-third of all neck injuries occur in frontal impacts [5]. Thus, neck injuries in frontal impacts warrant detailed research.

Crash tests are important for reducing injuries in accidents, but the testing method has limitations. A 50th percentile adult male dummy in a normal sitting posture exposed to standardized crash scenarios is commonly used in automobile crash safety tests. Even in the same crash situation, there are substantial differences between impact tests and a real accident. The dummies used in vehicle crash simulations are often stiffer than real human bodies and respond differently $[6,7]$. Particularly in the soft and flexible cervical 
and spinal regions, dummies are typically unrealistically stiff and unlikely to have the same compliant response to human bodies [8]. In the real world, drivers tend to act on the brake pedal and/or steering wheel to avoid or prepare for an upcoming impact [9-12]. However, a dummy cannot perform evasive maneuvers before impact. Thus, cars that achieve the highest scores in crash tests are optimized for protecting dummies, rather than human occupants.

With increasing computational power and progression in human body biomechanics, human body modeling (HBM) is widely utilized in the field of crash simulation. HBM involves computational modeling of human physiological structure and can be applied to study the influence of muscular activity and post-fracture response.

Studies of the effect of braced action on vehicle impact have been undertaken using a combination of experimental testing and numerical analysis with active HBM. In these tests, volunteers are typically subjected to low acceleration in sled devices while physiological signals are recorded. In numerical analysis, HBM with experimental parameters is employed in crash injury research. The investigation of muscular effects on cervical injuries can be conducted using the same research paradigms.

Choi et al. [13] employed volunteers in sled tests, measuring reaction forces on the steering wheel and brake pedal, as well as the activities of muscles in the upper and lower extremities. Computational analysis using a human finite element (FE) model with muscle activities obtained from an experiment in a similar environment was conducted and validated by experimental results [13]. Using a similar method, Ejima et al. [14] compared the behavior of relaxed and braced occupants in a pre-crash scenario. It was found that the neck and abdominal muscles were the most highly activated and kinematics of the head-neck-torso were strongly influenced by the muscle activity. To extend understanding of whiplash injury, Kumar et al. conducted sled tests using volunteers to determine the recruitment of cervical muscles with varied magnitudes of low velocity, different sitting positions under conditions of awareness and unawareness of upcoming frontal and rear-end impacts. Electromyography (EMG) signals of cervical muscles were found to exceed the maximum voluntary contraction (MVC) level, and excessive contraction of the muscles could contribute to injury [14-16]. To protect volunteers from injury, the sled test is limited to low-speed impact, meaning that responses of occupants in complicated high-speed accidents cannot be elicited using this method.

Reconstructing a virtual collision scenario in a driving simulator provides an alternative method. Virtual simulations could be an effective tool for examining realistic avoidance behavior of occupants in an imminent crash. Autrey et al. [17] designed a driving task involving a sudden frontal crash in a driving simulator, while the driver's actions were recorded by cameras throughout the driving period. The driving action was used in a numerical simulation of a frontal crash. Thus, a driving simulator method was used to investigate driving behavior and evasive maneuvers in an emergency situation. However, muscle activity, which could reveal additional information about human action, was not recorded in the study mentioned above.

The current study sought to investigate contraction of drivers' cervical muscles in a vehicle crash scenario and to examine the effect of neck muscle strength on collision injury, at low and high speeds. The study was composed of two parts. One part reported a driving simulator study designed to determine the cervical muscle activation in an unavoidable impact. Frontal impacts at different velocities were reconstructed in a driving simulator. EMG signals from selected cervical muscles during impact were recorded and analyzed. The other part reported a numerical simulation to study the effect of muscle force on injury outcome. The averaged muscle activations were applied to an active cervical HBM using MADYMO software.

\section{Experimental Study of Cervical Muscle Activity During Emergency Braking}

The experiments were performed in a driving simulator at the State Key Laboratory of Automobile Simulation and Control, Jilin University, China (Fig. 1). A sudden frontal impact was replicated in the simulator.

\subsection{Experimental Setup}

The driving simulator had the capacity to perform yaw, pitch and roll motion and was equipped with a realistic control

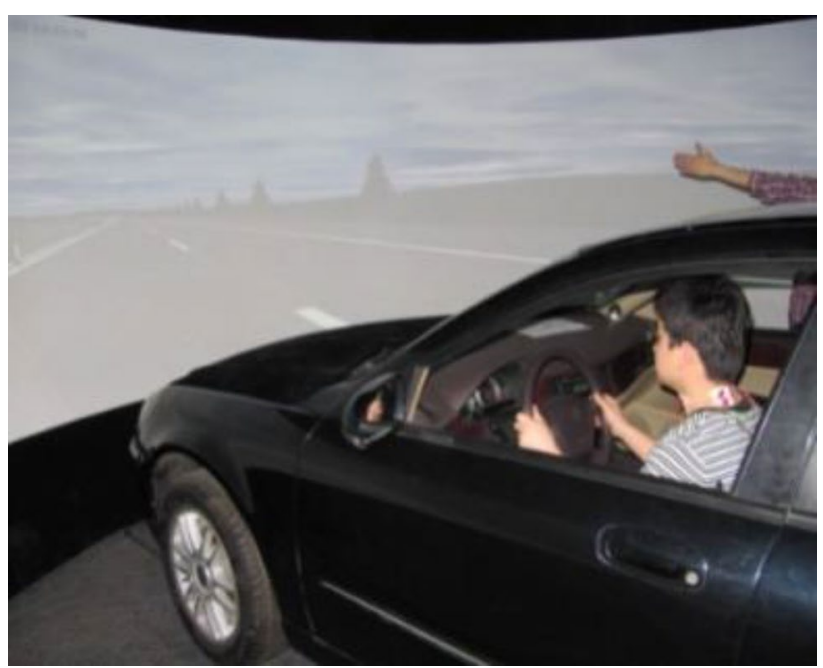

Fig. 1 Driving simulator 
Turning Point obstacle car

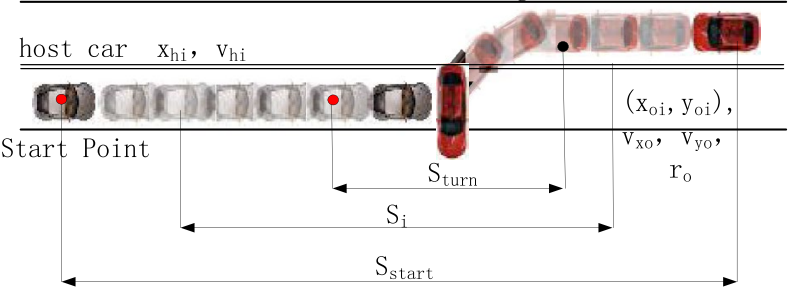

Fig. 2 Simulated crash scenario

Table 1 Volunteer data

\begin{tabular}{lrrrlr}
\hline & Age & Height $(\mathrm{cm})$ & Weight $(\mathrm{kg})$ & $\begin{array}{l}\text { Sitting } \\
\text { weight } \\
(\mathrm{cm})\end{array}$ & BMI $\left(\mathrm{kg} / \mathrm{m}^{2}\right)$ \\
\hline Mean & 28 & 174.1 & 67.4 & 91.8 & 22.5 \\
SD & 3.2 & 4.1 & 3.8 & 3.3 & 1.2 \\
\hline
\end{tabular}

operation system. The steering wheel and brake pedal induced a sensation similar to that of normal real-world driving.

The driving scenario is shown in Fig. 2. The volunteer drove the host car in one lane at a specified speed. One obstacle car, which crossed into the other lane, suddenly cut into the participant's lane and stopped 10 meters in front of the host car.

\subsection{Selection of Volunteers}

The selection of volunteers was based on the following criteria: similar body size to 50 percent American male and no history of cervical spine pain in the past 12 months. The mean age, height and weight of the sample were 28 (SD 3.2) years, 174.1 (SD 4.1) $\mathrm{cm}$ and 67.4 (SD 3.8) kg. (Table 1)

The protocol of the experiments was reviewed and approved by the Jilin University Ethics Committee, and all volunteers submitted their informed consent in a document that complied with the Helsinki Declaration.

\subsection{Selection of Muscles of Interest}

There are numerous muscles on the cervical spine, including small, intersegmental muscles and larger muscles that originate from the skull and insert to the thorax. The sternocleidomastoideus (SCM), trapezium (TRP) and splenius cervicis (SPL) were selected as muscles of interest. In the neural position, the SCM, SPL and TRP have the largest moment-generating capacity in flexion, extension and axial rotation [18]. These large surface muscles were selected as muscles of interest because of their large force-generating capacity and the ease of measurement with EMG.
The main actions of the SCM include forward flexion of the head on the trunk when acting bilaterally. The TRP is capable of extending the head. The SPL extends the head and neck as well as assists in rotation.

\subsection{Test Procedure}

The corresponding magnitude of EMG in maximum voluntary contraction (MVC) root mean square (RMS) was measured and used in the normalization of EMG signals. EMG data collected during MVC and impact simulation were subjected to quantitative and statistical analysis.

The EMG was recorded using a MP150 physiology recorder produced by BioPac Systems, Inc (California, USA). The sample rate was set to $1 \mathrm{kHz}$. Proper electrode placement and locations were suggested by the SENIAM project [19]. The skin was shaved, wiped with 50/50 alcohol/distilled water and coated with electrode gel. The electrodes were placed bilaterally on the most prominent part of the SCM and the SPL at the C4 level. A ground electrode was placed above the right acromion.

The MVC was conducted approximately $30 \mathrm{~min}$ before the formal tests. The volunteers were asked to sit on the chair with arms perpendicular to the trunk to reduce the involvement of the biceps and deltoid, feet suspension to avoid lower extremity muscle force. The methods to elicit the MVC of the muscles were as follows: For the TRP, the volunteer shrugged the shoulder with maximum force; for the SCM, the volunteer performed left and right lateral flexion; and for the SPL, the volunteer performed flexion and extension of the head. The EMG amplitudes recorded during the simulation trials were normalized against these maximal values.

In the driving simulator, volunteers drove the simulated vehicle at speeds of $20 \mathrm{~km} / \mathrm{h}, 50 \mathrm{~km} / \mathrm{h}, 80 \mathrm{~km} / \mathrm{h}$ and $100 \mathrm{~km} / \mathrm{h}$. The EMG signals of selected muscles were recorded.

EMG signals in the MVC test and simulation were subjected to band-pass filtering of $10-350 \mathrm{~Hz}$, full wave rectified with a linear envelope.

RMS reflects the average level of muscle discharges over a period of time and is considered to be related to the number of motor units recruited, expressed as

$\mathrm{RMS}=\sqrt{\frac{1}{T} \cdot \int_{0}^{T} \operatorname{EMG}^{2}(t) \mathrm{d} t}$

where $\operatorname{EMG}(t)$ is amplitude of signal and $T$ denotes the length of the signal. RMS can be used to estimate the muscle strength produced by a muscle. The EMG amplitude corresponding to MVC for each muscle was given a value of 
Fig. 3 50th percentile male facet neck model (a) front view; (b) side view; (c) rear view

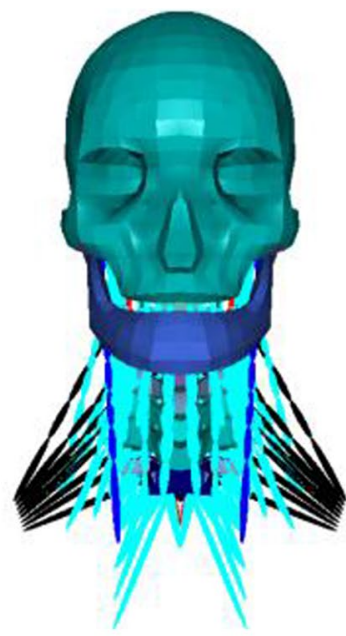

$\mathbf{a}$

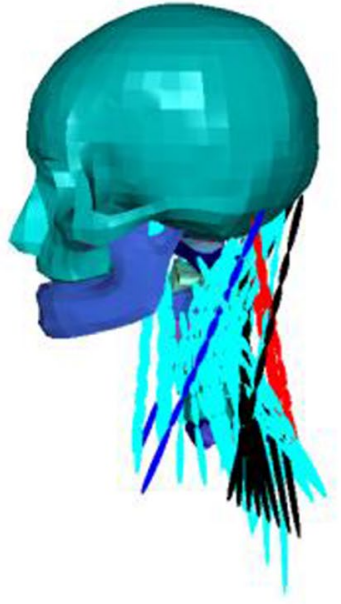

b

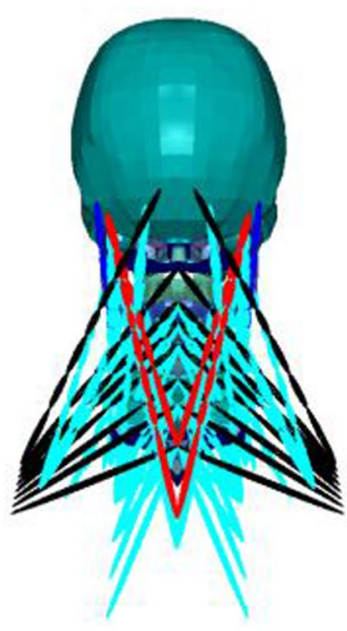

c
$100 \%$. The EMG amplitudes recorded in the simulation test were normalized against these maximal values.

In addition, the slope of EMG is expressed as follows:

$$
\text { Slope }_{\mathrm{EMG}}=\left(\mathrm{EMG}_{\mathrm{max}}-\mathrm{EMG}_{\mathrm{act}}\right) / T
$$

where $\mathrm{EMG}_{\max }$ is the peak value of $\mathrm{EMG}, \mathrm{EMG}_{\mathrm{act}}$ is the activation of EMG and $\mathrm{T}$ is the time of EMG signal raising from activation state to peak value. The time of muscle activation was determined by an EMG increase of 5\% of MVC over the baseline.

Statistical analysis was carried out using the SPSS statistical package to calculate descriptive statistics, correlation analysis between EMG and driving speed.

\section{Numerical Study of Frontal Impact}

In a numerical study of impact injury, muscular activation values were based on the data collected in the experimental study.

\subsection{Human Body Model}

A MADYMO 50th percentile male facet occupant model with active neck muscles was used in the simulation.

The facet occupant model is composed of chains of rigid and flexible bodies, which represent the inertial properties of the segments, connected by kinematic joints. The outer surface is described with meshes of shell-type contact element, representing the deformation of soft tissues defined by stress-based contact characteristics. The model allows a more accurate geometric representation and efficient computation.

The facet neck model designed for muscle tensing simulation is defined with relevant muscles addition to anatomical structures, as shown in Fig. 3. In the figure, the SCM, TRP and SPL are shown in blue, black and red. Most muscles are represented by more than one Hill-type muscle element to account for different attachment points of the muscle group. In this model, 16 pairs of cervical muscles are modeled by 68 symmetrical pairs of muscle elements. The muscle elements are divided into segments that curve around the vertebrae. The muscle activation can be defined by a time-varying function.

\subsection{Simulation of Braced Occupant}

The steering wheel and airbag typically make forceful contact with the occupant during a secondary collision. Thus, their settings and arrangement can have a significant effect on injury. As such, these factors were excluded in the simulation to reduce the interference of external factors.

The occupant model in driving posture was positioned in a sled system equipped with a seat, floor panel and belt. Sliding contact interfaces were defined between the seat and the skin of the model. The three-point belt system was set with standard belt stiffness and retractor properties.

The activity of muscles was set by a time-dependent curve, where the experimental slope was also considered. The simulations were conducted in two groups for each speed. The deceleration pulses were acquired from frontal $100 \%$ rigid barrier impacts at speeds of 20, 50, 80 and $100 \mathrm{~km} / \mathrm{h}$. In the passive occupant model, all activations of cervical muscles were set as 0.005 for model stability. In contrast to the passive model, activations of the SCM, TRP and SPL muscles in the active model were set according to the recorded experimental activation values. The whole simulation process took $250 \mathrm{~ms}$, and the muscle activities and impact pulse were released at $0 \mathrm{~ms}$ and $100 \mathrm{~ms}$, respectively. This left $100 \mathrm{~ms}$ for the muscles to react to the upcoming 
impact, because it takes time for muscles to generate muscular forces that can be transformed to joint movements after contraction.

In accord with the Euro NCAP requirements, neck shear, tension and extension were used as criteria to assess neck injuries. These parameters during impact were selected for injury analysis.

\section{Results of the Experimental Study and Numerical Study}

Nine of ten volunteers pressed the brake pedal with their right foot, gripped the steering wheel with both hands, stretched their elbows and pushed their trunk to the seatback when the obstacle vehicle cut in front of the host vehicle. The head had a slight backward tendency due to this movement, consistent with the evasive action of passengers described in previous studies [14]. One volunteer had a habitual evasive action of pressing the right foot to the brake pedal and rotating the steering wheel to avoid the obstacle at the same time. The data for this volunteer were removed from the analysis.

\subsection{EMG Amplitude of Cervical Muscles}

The normalized RMS of EMG (\%RMS) was used to characterize the muscle force produced during the collision. The mean RMS of the three muscles of the SPL, SCM and TRP was calculated for nine volunteers. The levels of muscle activation are shown in Fig. 4. The SPL had the lowest magnitude of normalized EMG (less than 13\%), while the TRP had the maximum activity (ranging from 18 to $26 \%$ ). The SCM and TRP exhibited similar amplitude patterns. With the increase in driving speed, the \%RMS tended to increase. This occurred because increased driving speed led

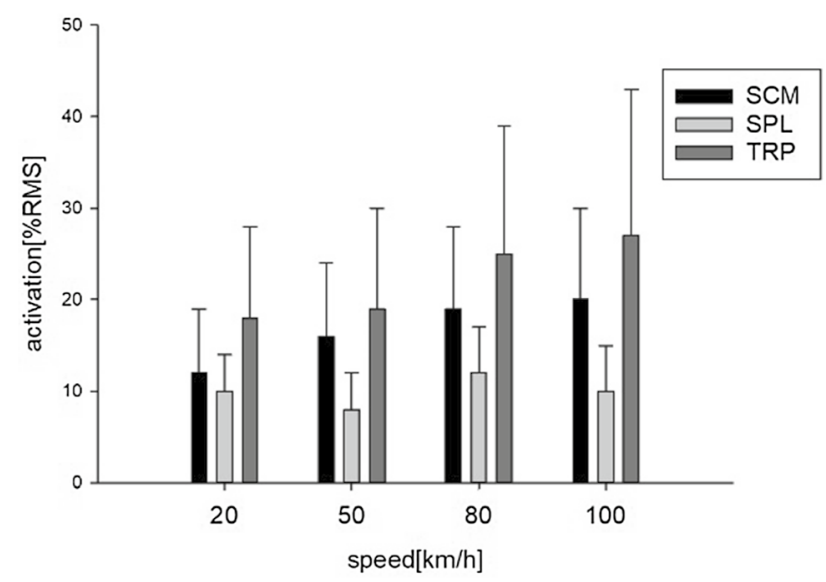

Fig. 4 Activation of cervical muscles

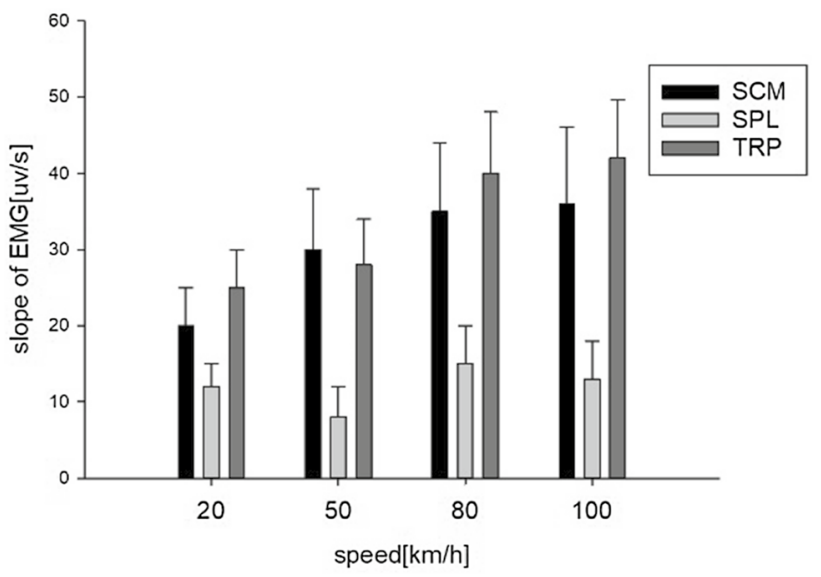

Fig. 5 Slope $_{\text {EMG }}$ of cervical muscles

to greater stimulation among volunteers, causing an increase in the level of muscle contraction. However, no regularity was found in the SPL.

The slope of EMG activity in the SCM and TRP increased with driving speed. The slopes of the EMG increase in the SPL did not follow the pattern exhibited in the SCM and TRP, as shown in Fig. 5.

\subsection{Statistical Analysis}

In a multivariate analysis of variance, the driving speed and the muscle examined had a significant effect $(P<0.01)$ on the EMG values. Increasing acceleration was associated with greater peak EMG. A least significant difference of the EMG demonstrated that the SPL was significantly different from the SCM and TRP $(P<0.001)$, while the SCM and TRP were similar to each other.

Statistical analysis of the slope of EMG exhibited a similar pattern of results to the EMG values. The driving speed and muscle examined also had a significant effect on the slope of the EMG $(P<0.05)$. The slopes varied for the examined muscles, and the SPL significantly differed from the other two muscles.

\subsection{Occupant Motion}

The drivers' motion during frontal impact at speeds of $20 \mathrm{~km} / \mathrm{h}$ and $50 \mathrm{~km} / \mathrm{h}$ is shown in Fig. 6. The start of the impact pulse was set as the beginning of collision. The sled was stopped by the impact pulse, while the occupant kept moving forward, and the movement was then restricted by the seat belt. The motion of the neck can be divided into protraction and flexion. From 0 to $60 \mathrm{~ms}$, the head exhibited horizontal translational displacement relative to the torso, which was called the phase of neck protraction motion. The neck was exposed to a high load when the terminal point 
a

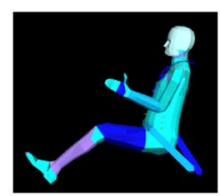

b

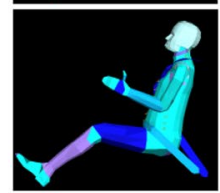

c

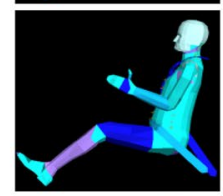

d

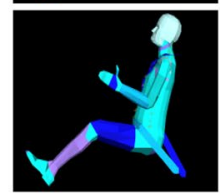

Oms
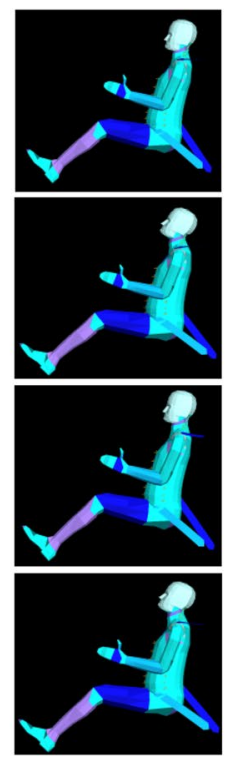

$30 \mathrm{~ms}$
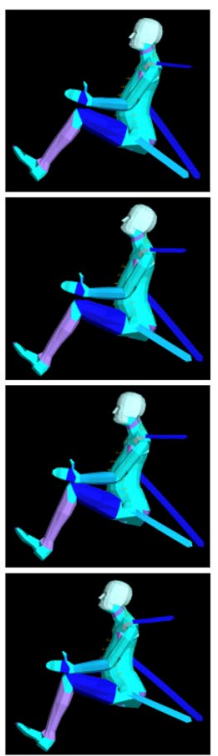

$60 \mathrm{~ms}$
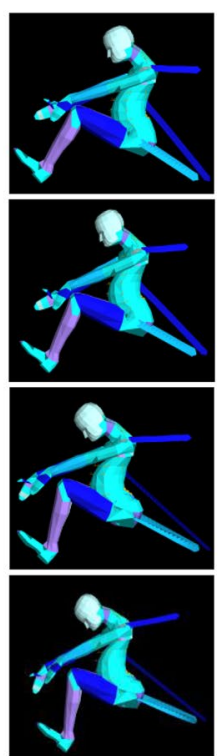

$90 \mathrm{~ms}$
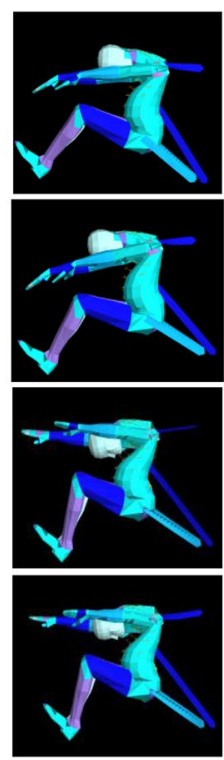

$120 \mathrm{~ms}$
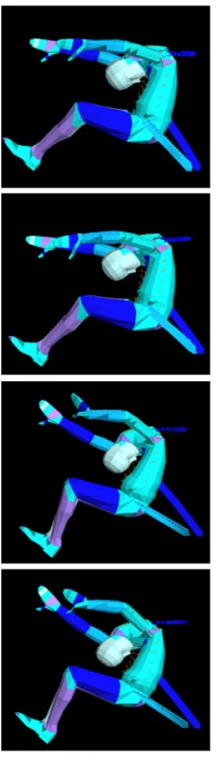

$150 \mathrm{~ms}$

Fig. 6 Motion during collisions in different muscle activation states and impact speed: a passive, $20 \mathrm{~km} / \mathrm{h} ; \mathbf{b}$ active, $20 \mathrm{~km} / \mathrm{h}$; $\mathbf{c}$ passive, $50 \mathrm{~km} / \mathrm{h}$; d active, $50 \mathrm{~km} / \mathrm{h}$

of neck protraction was reached. This led to the phase of neck flexion motion, which was observed from $90 \mathrm{~ms}$. The head, restrained by the neck, was moving downward. Comparing the motion of active and passive cervical occupants in frontal impacts of the same speed revealed differences in the trajectories of the head. In the phase of neck protraction motion, the head extended backward due to muscle force in the braced occupant, while the head remained upright in the relaxed occupant. In the phase of neck flexion motion, the head was retracted by the torso through the cervical spine. At $90 \mathrm{~ms}$, the head of the active occupant model had a slightly more forward position than the passive model. At $120 \mathrm{~ms}$ and $150 \mathrm{~ms}$, there were no differences between the two models. In addition, the results revealed that there were no differences in the trajectory of other segments.

\subsection{Injury Parameters}

The injury parameters of the cervical muscles, with muscles in an activated or relaxed condition, are shown in Fig. 7.

The main peak value of the loads was observed in both active and passive models. The peak value for all the parameters increased with impact speed. The load reached its peak at approximately $70 \mathrm{~ms}, 65 \mathrm{~ms}, 60 \mathrm{~ms}$ and $50 \mathrm{~ms}$ at speeds from 20 to $100 \mathrm{~km} / \mathrm{h}$, respectively. The active model was less stressed than the passive model in the 20 and $50 \mathrm{~km} / \mathrm{h}$ impact conditions. In high-speed impacts, some parameters for the active model had high values. At the same time, because all of the loads started earlier in the active model, it can be assumed that the active model had small change rates of loads.

In the $20 \mathrm{~km} / \mathrm{h}$ impact condition, the maximum torque reached $16.8 \mathrm{~N} \cdot \mathrm{m}$ and $27 \mathrm{~N} \cdot \mathrm{m}$ in the active model and passive model, respectively, and the maximum shear values were $1583.4 \mathrm{~N}$ and $1941 \mathrm{~N}$. The active model exhibited greater tension in the neck than the passive model, by $34 \mathrm{~N}$, although the incline of tension was smaller.

For the $50 \mathrm{~km} / \mathrm{h}$ simulation, the torque reached 20.3 $\mathrm{N} \cdot \mathrm{m}$ and $33.4 \mathrm{~N} \cdot \mathrm{m}$ for the active and passive model, respectively. The shear reached $1952 \mathrm{~N}$ and $2420 \mathrm{~N}$, respectively. The peak tension in the active and passive model was $921 \mathrm{~N}$ and $839 \mathrm{~N}$. A second peak was observed at approximately $130 \mathrm{~ms}$, and the peak values decreased compared with their counterpart in various degrees.

When exposed to high-impact pulses, the soft tissue performed poorly in terms of computational stability. The curves of tension and shear were completely different from those in the lower-speed condition. The active model exhibited greater tension force and shear force, while the passive model exhibited greater torque. In the $80 \mathrm{~km} / \mathrm{h}$ impact condition, the second peak of tension and shear was greater than the first peak. In the $100 \mathrm{~km} / \mathrm{h}$ impact condition, three peaks of a similar magnitude were observed in the tension curve. The first peak of the shear curve was much higher than the second peak. 


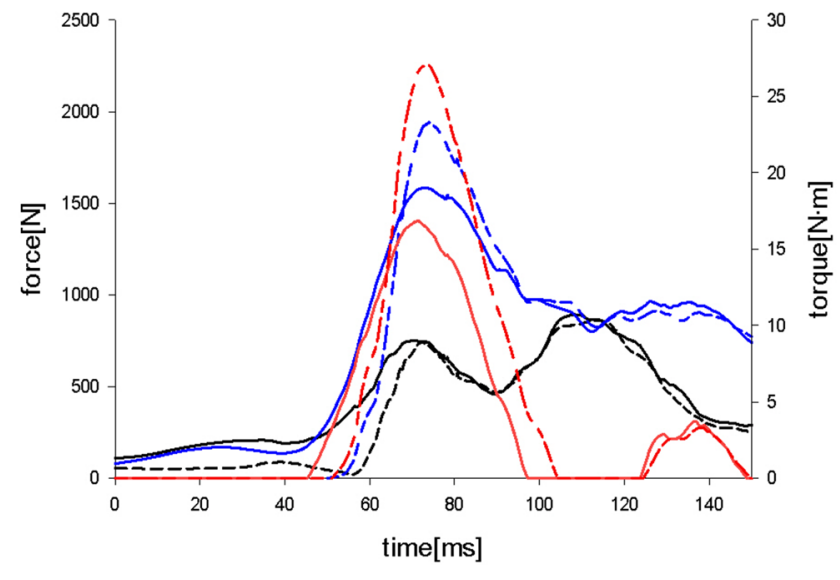

a

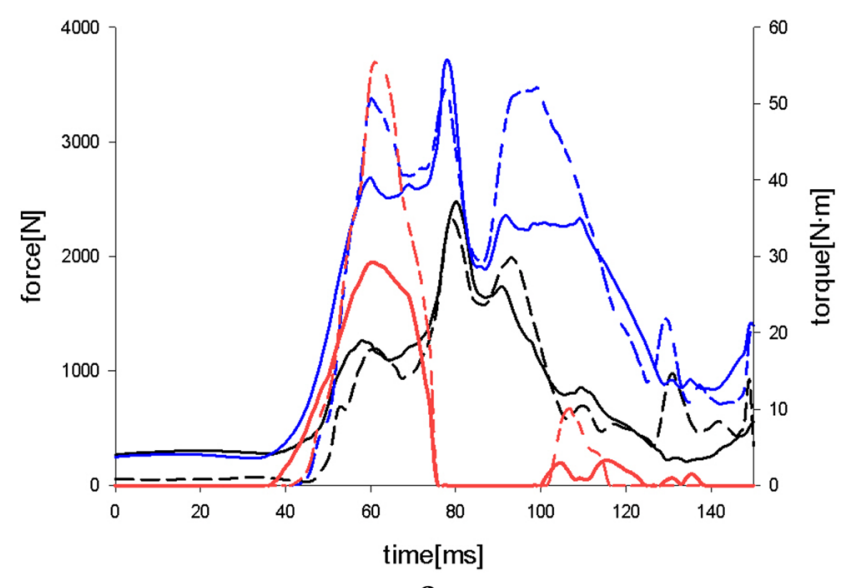

c

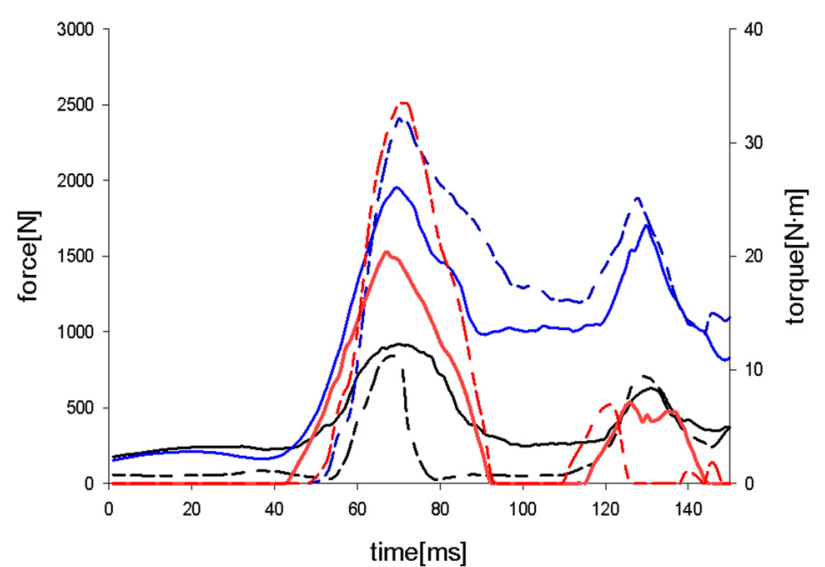

b

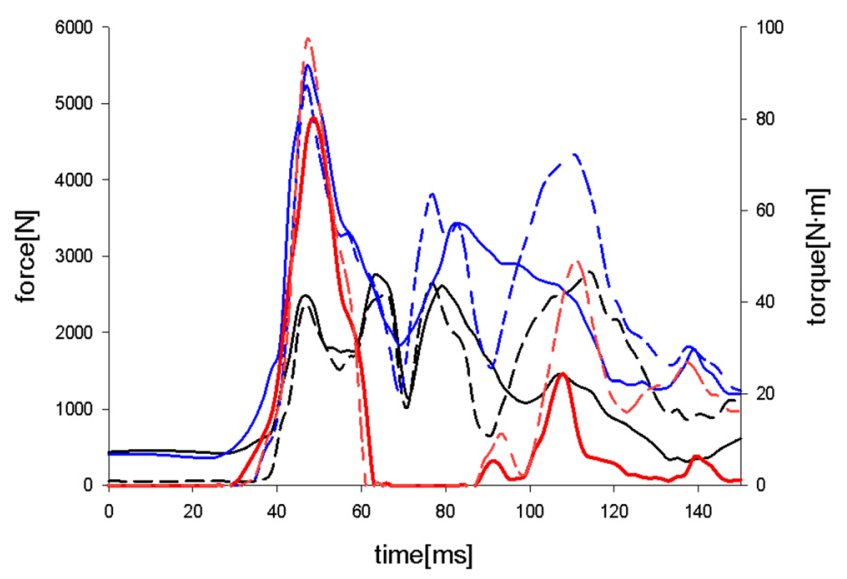

d

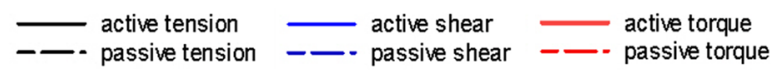

Fig. 7 Injury parameters in frontal impact: a $20 \mathrm{~km} / \mathrm{h}$; b $50 \mathrm{~km} / \mathrm{h}$; $80 \mathrm{~km} / \mathrm{h}$; d $100 \mathrm{~km} / \mathrm{h}$

\section{Discussions}

Considering the differences between crash tests and realword traffic accidents, it is critical to understand how closely simulated injuries conform to real-world injuries. The key issue relates to the investigation of occupantinitiated muscular activation and its effects on impact responses and occupant behavior during vehicle collisions.

Investigation of the reaction of occupants in low- to high-speed realistic impacts is challenging, because the sled test using human volunteers is typically limited to low-speed impacts for ethical reasons, constraining the realism of the driving environment. Therefore, reconstructing impact scenarios using a dynamic driving simulator could provide a powerful tool for investigating occupants' reactions in traffic accidents. HBMs that consider muscle activity are widely employed in studying muscle activity in crash scenarios.
In the current study, volunteers participated in driving simulators to investigate muscular responses of drivers' cervical muscles in a simulated frontal impact. A scenario of sudden unavoidable frontal impact was used, in which volunteers undertook simulated driving at speeds of 20,50 , 80 and $100 \mathrm{~km} / \mathrm{h}$.

The normalized EMG revealed that the maximum activity for the TRP ranged from 18 to $26 \%$, while the activation of SCM varied from 12 to $22 \%$. The SPL exhibited the lowest level of normalized EMG activity, which was less than $13 \%$. The activation of the cervical muscles increased with driving speed. The SCM and TRP exhibited similar activation and differed from the SPL.

According to the anatomy of the human neck, the main actions of the SCM include forward flexion of the head on the trunk when acting bilaterally. The TRP is capable of extending the head. The SPL extends the head and neck as well as assisting in rotation. The SPL and TRP are agonist 
muscles for the extension of the head, while the SCM is the antagonist muscle. During the collision, the activated muscles change the kinematics of the occupant, typically causing the head to stretch backward. The SPL, which acts as an agonist muscle for the extension, is less activated than the SCM, which is the antagonist muscle. The introduction of the steering wheel provides an important constraint for the driver besides the seat and seat belt. With the anticipation of an unavoidable upcoming crash, the driver tends to extend the elbow and knee joints, pushes the trunk to the seatback and moves the head backward with the trunk [14]. The whole body muscles, especially in upper extremities and trunk, share the role of the SPL. Thus, the extension of the head is not only caused by the cervical muscles. At the same time, the brake pedal provides support to the leg, which also affects the whole body kinematics.

In the second part of the current study, a numerical study was carried out with an active cervical muscle model. Two key points should be noted. First, the muscle force was relatively small compared with the impact pulse; second, the duration of impact pulse was approximately $150 \mathrm{~ms}$, and the movement of the segment was $50-150 \mathrm{~ms}$ after the activation of the muscle [20]. Thus, the active muscle force has traditionally been ignored in crash safety. The current study concentrated on the pre-crash reaction, including the muscle activation released before the impact pulse in the simulation. The muscle force had sufficient time to act and influence the kinematics of the occupant.

In terms of the trajectory of the models in different impact pulses, the head of the active model stretched backward before the pulse was transmitted to the human model, while no change was observed in the passive model. The head of the active model reached the end of the natural range of protraction earlier than the passive model. The motion of the other segments was not affected.

In terms of the neck loads for different speeds of frontal impact, the tension, shear and torque were relevant to the neck injury criteria. Activation of the cervical muscles appears to be a positive factor in reducing neck injury in lower-speed frontal impacts. The peak torques and shears in the active model were much lower than those in the passive model. The active model exhibited slightly greater neck tension than the passive model, but lower slope. In highspeed frontal impact, the inclusion of neck muscle activation reduced the torque but enhanced the shear, which could increase the risk of shear injury.

The muscle itself is one of the neck injury sites. The mechanism of cervical muscle injury involves the lengthening of the muscle by the inertia of the head during active contraction. The procedure is called eccentric contraction. In frontal impact, the posterior located muscles are activated and lengthened in the phase of protraction motion and flexion motion. Thus, the TRP and SPL would be likely to sustain more serious injury in the active model than the passive model.

One limitation of the current study is related to the absence of activation of other cervical muscles. The human neck has complex musculoskeletal anatomy with redundancy. All muscles are involved in movement, in accord with muscle recruitment strategies. With EMG measurement alone, it is not practical to acquire the activation scale for each muscle. In future, estimation of activation of cervical muscles based on measured activation should be studied.

\section{Conclusions}

Driving simulators provide an effective tool for studying the evasive behavior of occupants in real traffic accidents.

Drivers often perform evasive maneuvers when they are aware of an impending collision. Evasive behaviors typically cause a significant increase in EMG activity compared with normal driving. The SCM and TRP muscles exhibit a similar pattern of activation.

Contraction of the cervical muscles can positively reduce the risk of neck injury in lower-speed frontal impact. However, the risk of shear injury may increase in high-speed impacts.

The posterior muscles experience eccentric contraction in frontal impact, potentially leading to muscle injury.

Acknowledgements This work is supported by National Natural Science Foundation of China (Nos. 51775236, 51675224, U1564214) and National Key R\&D Program of China (Nos. 2017YFB0102600, 2018YFB0105205).

\section{Compliance with Ethical Standards}

Conflict of interest On behalf of all the authors, the corresponding author states that there is no conflict of interest.

Open Access This article is distributed under the terms of the Creative Commons Attribution 4.0 International License (http://creativeco mmons.org/licenses/by/4.0/), which permits unrestricted use, distribution, and reproduction in any medium, provided you give appropriate credit to the original author(s) and the source, provide a link to the Creative Commons license, and indicate if changes were made.

\section{References}

1. National Highway Traffic Safety Administration.: Traffic safety facts 2015: summary of motor vehicle crashes. https://crashstats .nhtsa.dot.gov/Api/Public/ViewPublication/812376. Accessed 8 Jan 2017

2. European Commission.: Mobility and transport: statistical pocketbook 2017. https://ec.europa.eu/transport/facts-fundings/Stati stics/pocketbook-2017_en. Accessed 8 Jan 2017 
3. Morris, A., Hassan, A., Mackay, M., et al.: Head injuries in lateral impact collisions. Accid. Anal. Prev. 27(6), 749-756 (1995)

4. Erbulut, D.U.: Biomechanics of neck injuries resulting from rearend vehicle collisions. Turk. Neurosurg. 24(4), 466-470 (2014)

5. Kullgren, A., Krafft, M., Nygren, A., et al.: Neck injuries in frontal impacts: influence of crash pulse characteristics on injury risk. Accid. Anal. Prev. 32(2), 197-205 (2000)

6. Crandall, J.R., Portier, L., Petit, P., et al.: Biomechanical response and physical properties of the leg, foot, and ankle. https://doi. org/10.4271/962424

7. Beeman, S.M., Kemper, A.R., Madigan, M.L., et al.: Occupant kinematics in low-speed frontal sled tests: human volunteers, Hybrid III ATD, and PMHS. Accid. Anal. Prev. 47, 128-139 (2012)

8. Svensson, M.Y., Ola, B., Davidsson, J., et al.: Neck injuries in car collisions: a review covering a possible injury mechanism and the development of a new rear-impact dummy. Accid. Anal. Prev. 32(2), 167-175 (2000)

9. Morris, R., Cross, G.: Improved understanding of passenger behaviour during pre-impact events to aid smart restraint development. In: International Technical Conference on the Enhanced Safety of Vehicles. No. 05-0320. Washington, USA (2005)

10. Hault-Dubrulle, A., Robache, F., Drazetic, P., et al.: Determination of pre-impact occupant postures and analysis of consequences on injury outcome Part I: a driving simulator study. Accid. Anal. Prev. 43(1), 66-74 (2011)

11. Begeman, P., King, A., Levine, R., et al.: Biodynamic response of the musculoskeletal system to impact acceleration. In: SAE International 24th Stapp Car Crash Conference. IEEE, IEEE Xplore Digital Library, pp. 3-33 (2007)
12. Klopp, G.S., Crandall, J.R., Sieveka, E.M., et al.: Simulation of muscle tensing in pre-impact bracing. In: International Ircobi Conference on the Biomechanics of Impact, Brunnen, Switzerland (1995)

13. Choi, H.Y., Sah, S.J., Lee, B., et al.: Experimental and numerical studies of muscular activations of bracing occupant. In: Proceedings of the 19th International Technical Conference on the Enhanced Safety of Vehicles. Washington, USA (2005)

14. Ejima, S., Zama, Y., Ono k., et al.: Prediction of pre-impact occupant kinematic behavior based on the muscle activity during frontal collision. In: Proceedings of the 21st Enhanced of Vehicles Conference, Stuttgart, Germany. Paper No. 09-0913 (2009)

15. Kumar, S., Narayan, Y., Amell, T.: Analysis of low velocity frontal impacts. Clin. Biomech. 18(8), 694-703 (2003)

16. Kumar, S., Ferrari, R., Narayan, Y.: An analysis of right anterolateral impacts: the effect of head rotation on the cervical muscle whiplash response. J. NeuroEng. Rehabil. 30(5), 536-541 (2005)

17. Hault-Dubrulle, A., Robache, F., Pacaux, M.P., et al.: Determination of pre-impact occupant postures and analysis of consequences on injury outcome Part I: A driving simulator study. Accid. Anal. Prev. 43(1), 66-74 (2011)

18. Vasavada, A.N., Li, S., Delp, S.L.: Influence of muscle morphometry and moment arms on the moment-generating capacity of human neck muscles. Spine 23(4), 412-422 (1998)

19. SENIAM.: Sensor location. http://www.seniam.org/. Accessed 10 Oct 2014

20. Wolf, S.D., Slijper, H., Latash, M.L.: Anticipatory postural adjustments during self-paced and reaction-time movements. Exp. Brain Res. 121, 7-19 (1998) 